\title{
Competency of Health Information Acquisition and Intention for Active Health Behaviour in Children
}

\section{Abstract}

Objective: To investigate the association between competency of health information acquisition, both online and offline, and the intention for active health behaviour in children.

Methods: This study was a population-based cross-sectional health survey utilising a two-stage random cluster sampling design conducted in a major city. Competency of health information acquisition was assessed by a rating scale designed specifically for this study. The intention for active health behaviour was measured by a vignettebased question. Data were analysed using multiple logistic regression modelling techniques with adjustment to the cluster sampling effect and potential confounding factors.

Results: After adjusting for potential confounding factors and the cluster sampling effect, intention for active health behaviour was significantly associated with competency of health information acquisition both online (OR=1.06, 95\%C.I.=1.01-1.12) and offline (OR=1.08, 95\%C.I. $=1.02-1.18)$.

Conclusions: Results suggested a positive relationship between competency of health information acquisition, both online and offline, and the intention for active health behaviour which have important public health implications on child health behaviour.

\author{
Lawrence T Lam, PhD ${ }^{1,2}$, \\ Mary KP Lam ${ }^{3}$
}

1 Department of Health and Physical Education, The Hong Kong Institute of Education, Hong Kong SAR.

2 Discipline of Paediatrics and Child Health, Sydney Medical School, The University of Sydney, Australia.

3 Faculty of Health Sciences The University of Sydney, Australia

Contact information:

Lawrence T. Lam.

Tel: (852) 29486685

Fax: (852) 29487848

Address: The Hong Kong Institute of Education. 10 Lo Ping Road, Tai Po, N.T. Hong Kong SAR, CHINA

$\equiv \mid$ Itlam@ied.edu.hk

\footnotetext{
Keywords

Health information seeking; Health behaviour; Intention; Children;

Epidemiology study; Survey
} 


\section{Introduction}

It has been well established that intention to perform health behaviour plays a central role in determining the actual performance of the health behaviour, as proposed in health behavioural models such as the Integrated Behavioural Model. [1, 2] Other determinants of health behaviour, also suggested in the model, include individual factors and other psychosocial factors. For individual factors, three main clusters of factors have been proposed including attitude towards the behaviour, social influence, and perceived control and self-efficacy.1 Of equal importance are other psychological factors that also have direct influence on the intention of the health behaviour and in turn the actual performance of the health behaviour. These include knowledge and skills for performing the target behaviour, salience of the behaviour, and environmental constraints. [1] The relationship between behavioural intent and the actual performance of the health behaviour has been demonstrated in studies of different areas ranging from smoking to blood donation. [311] For example, in the study by Tzilos et al, it was found that the intention to quit smoking was a significant and strong predictor of the actual quitting behaviour even in populations of young people with psychiatric problems. [7]

As knowledge and skills for performing the target behaviour are influential to the intention for health behaviour, a logical question to ask is how would an individual be able to acquire the appropriate health knowledge and skills? In this age of new media, access to information and the individual's ability to access information are the keys to the target knowledge and, to a certain degree, to skills. [12] In other words, the competency of information acquisition is a fundamental attribute of an individual for assessing information and thus the key to knowledge and skills.

Adopting the definition commonly used in the area of human performance studies, 'competency' refers to characteristics, used singularly or in combi- nation, which result in successful performance. [13] These characteristics include content knowledge, specific skills, mindsets, thought pattern, and the like.[13] In other words, competency can be viewed as the "knowhow", not only in the sense of skills, but also in the individual's mentality. Applying this concept to health information acquisition, the competency of health information acquisition can be defined as the "knowhow", in the full sense, of knowing how to, as well as having the skills to, acquire specific health information. While knowledge and skills of specific health behaviour have been theorised to have a direct effect on the intention for health behaviour, it could also be argued that competency of information acquisition, as an essential precursor for health knowledge and skills, could also have an effect on behavioural intent. In other words, there could be a relationship between competency of information acquisition and the intention of health behaviour. This theoretical conceptualisation can be applied to the area of child health, particularly in the area of healthy lifestyles that require motivation for behavioural change, such as the reduction of unhealthy food intake and greater involvement in physical activities. It can be hypothesised that the competency of information acquisition of young people for a certain health behaviour, either online or offline, could have a relationship with their intention for the behaviour.

The aim of this study is to examine the association between the competency of health information acquisition of children, both online and offline, and their intention for health behaviour. It is hypothesised that there is a positive relationship between the competency of young people's health information acquisition and their intention for active health behaviour.

\section{Methods}

This study was a population-based cross-sectional health survey utilising a two-stage random cluster 
sampling design. The study was conducted in a major city in the Guangxi Province of the South Western region of China in November 2012. The city is the most populated city of the Province with an estimated population of about 6.7 million in 2010. The population size for young people aged between 15 and 19 years was estimated to be 505,677 . This represented about $7.6 \%$ of the total population in the city. Institute ethics approval for the study was granted by the Human Ethics Committee of the Hong Kong Institute of Education.

The sample consisted of school students aged between 12-16 years with the total student population attending junior high schools in the designated region as the sample frame. The local education department provided a list of junior high schools located within the boundary of the school district for sampling. The sample was generated using a two-stage random cluster sampling technique. First, using individual schools as the primary sampling unit, a number of schools were randomly selected with a probability proportional to the size of the target population. Second, using the class as the secondary sampling unit, different clusters of students were randomly selected from each grade of the selected schools. Participates were recruited from four high schools and 36 different classes.

The health survey was conducted within two weeks on campus at different schools. Parents and students from the selected classes from different schools were informed of the survey with a written information letter. They were invited to participate in the study and wilful consent was implied by the filling in of the questionnaire. During the survey students were asked to fill in a self-reported questionnaire designed specifically for the study.

Competency of health information acquisition was measured by the Competency of Health Information Acquisition Scale (CHIAS) which was specifically designed for this study. The conceptualisation of the competency of health informa- tion acquisition was based on the definition and working model of competency commonly used in the area of human performance. [14] The items for the initial CHIAS were formulated based on the suggestions of an expert panel and items found in other instruments that might contain a domain of competency of health information seeking for a specific health condition, such as the eHealth Literacy Scale (eHEALS).[15] Items in the bank were then further screened by a panel of experts for their suitability to be included in the initial CHIAS. Unsuitable items were removed from the pool. As a result 12 items were included in the new instrument. As a self-reported instrument, the items were formulated as questions asking individuals to respond with a Likert scale rating from 1 to 4 (1=Totally disagree, 4=Totally agree). An example item was: "I know how to obtain the health-related information I need for the specific condition on the Internet". These items were then subjected to the Kaiser-Meyer-Olkin (KMO) Measure of Sampling Adequacy test and the Bartlett's Test of Sphericity resulting in a $\mathrm{KMO}$ index of 0.785 and significant Bartlett's test $(\chi 228=2166.38$, $p<0.0001)$ suggesting that an Exploratory Factor Analysis (EFA) was appropriate. Items were then subjected to the EFA with the principal component method used for covariance structure analysis with an Eigene value $>1.0$ for acceptance of factors. A factor loading value of 0.45 was used as selection criteria for retention of items. The EFA yielded a 2-factor structure with $53.2 \%$ of variance explained. These factors were labelled as "Online Health Information Acquisition" and "Offline Health Information Acquisition" according to the nature of items with 4 items in each factor. To determine internal reliability, Cronbach's alpha coefficients for each subscale and correlations between subscale and total were calculated. Results indicated a moderately high reliability of the total, online, and offline scales with Cronbach's alpha of 0.76 , 0.74 , and 0.64 respectively. Correlations between 
online and offline subscales and the total scale were 0.840 and 0.845 with a moderately weak correlation of 0.42 between the two. For analysis, the summative scores for each subscale were calculated to be the online CHIAS and offline CHIAS scores.

Intention for active health behaviour was assessed using a vignette-based question asking the respondent to imagine that he/she might have a condition identical to what was described in the CHIAS. The respondent was then asked to provide the actual health-related action he/she was going to take. According to the nature of the responses, they were then classified as an intention for active health-related behaviour or some other categories of non-active behaviours.

Information collected in the survey included demographics, whether the respondent was a single child, their parental education levels, and whether the respondent was living with parents, and ownership of a computer. Data on risky health behaviours, including drinking, smoking, and sedentary behaviour were recorded using a frequency and duration exposure method for quantify moderately rigorous activity involvement. The WHO recommends 60 minutes of moderate-to-vigorous daily physical activity for young people aged 5-17 years, as such a level less than half of the recommended level was considered as sedentary in this study.16 Familial information was also collected including respondents' parental risky behaviours, namely parental drinking and gambling, as well as parental health status in the last 3 months. Health literacy was assessed using the short form of the Test of Functional Health Literacy for youth (s-TOFHLAd) translated into the Chinese language and validated by Chang et al. $[17,18]$ It consists of 36 items in a series of two health-related reading passages. In each passage, words are deleted from the original text and respondents must fill in the blank by selecting an appropriate word from a list of four possible choices. Results from a valida- tion study suggested good reliability and validity with a Cronbach's alpha of 0.85 and a test-retest coefficient of 0.95 with one week interval. For ease of analysis, the raw scores were categorised into three levels of health literacy, low, moderate, and high, in accordance to the cut-off points of $<25,25-31$, and 32 or above as suggested by Chang.[19]

Data were analysed using the Stata V10.0 statistical software program. Since the study was of a cluster sampling design, data were set up with the survey design function utilising the svy commands for handling the cluster sampling effect. Descriptive analyses were conducted with mean and standard deviation (s.d.) for the online and offline CHIAS scores or percentages for categorical variables. Bivariate analyses were conducted to examine the unadjusted relationships between competency of health information acquisition, variables of interest, and intention for active health behaviour. The Analysis of Variance approach (ANOVA) was used for analysing the online and offline CHIAS scores and Chi-squared tests for categorical variables with adjustment to the cluster sampling effect. Further multivariate analyses were conducted using multiple logistic regression modelling techniques with adjustment for the cluster sampling effect and potential confounding factors identified in the bivariate analyses. Separate logistic regression analyses were conducted to examine the association between the online CHIAS and the intention for active health behaviour, as well as the relationship between the offline CHIAS and the intention for active health behaviour. All significant variables identified in the bivariate analysis were included in the analysis. A significance level of $5 \%$ was used for hypothesis testing. Frist degree interaction terms of potential confounding variables were also tested with a significance level of $1 \%$. 


\section{Results}

A total of 1035 students responded to the survey providing usable information. The sample consisted of children aged between 12 and 16 years old with slightly more than half aged 14 years or older $(n=722,55.3 \%)$ and a mean age of 14.2 years (s.e. $=0.14)$. There were slightly more males $(59.7 \%)$ than females. In terms of demographics, the majority of the respondents were living with their biological parents $(n=1142,87.5 \%)$. Slightly more children had siblings than single children ( $n=595$, $45.6 \%$ ), with the majority of their parents attaining at least a level of secondary education with about $11 \%$ of fathers and $8 \%$ of mothers receiving post secondary education levels including university and post graduate education. About $8 \%(n=99)$ indicated that they were ex-smokers or was still smoking at the time of survey, and 551 (42.2\%) reported that they had consumed alcohol at least once in the three last months prior to the survey. For physical activity involvement, the majority ( $n=994,76.2 \%)$ could be classified as sedentary.

In terms of parental health conditions and health behaviours, 384 (29.4\%) of the respondents iden- tified that either their father or mother had consumed alcohol to the extent of being drunk in the last 3 months prior to the survey and 186 (14.3\%) reported that their parents had gambled in the last 3 months. About a quarter $(n=338)$ reported that one of their parents was ill in the last 3 months and nearly $11 \%(n=141)$ indicated that both parents were ill in the past 3 months. For health literacy levels, the scores of $628(48.1 \%)$ respondents could be classified as high, 558 (42.8\%) moderate, and $119(9.1 \%)$ low.

For the competency of health information acquisition, respondents scored a mean of $12.2($ s.d. $=2.69)$ for the online CHIAS and a mean of 10.8 (s.d.=2.73) for the offline CHIAS with a possible total score of 16 for each subscale. In terms of the outcome variable, 30\% ( $n=391)$ of the respondents indicated an intention for active health behaviour to the vignette question.

The bivariate relationships between competency of health information acquisition, demographics, familial variables, personal risky health behaviour, parental health problems, health literacy, and intention for health behaviour were examined. The results were summarised in Table 1. As shown,

Table 1. Frequency (\%) and mean (s.e.) of competency of health information acquisition, other variables of interest, by intention for active health behaviour and their unadjusted associations. ( $N=1035)$.

\begin{tabular}{|c|c|c|c|}
\hline & \multicolumn{2}{|c|}{ Intention for Active Health behaviour } & \multirow[t]{2}{*}{ Results * } \\
\hline & Yes $(n=391)$ & No $(n=914)$ & \\
\hline \multicolumn{4}{|c|}{ Demographics } \\
\hline \multicolumn{4}{|l|}{ Sex } \\
\hline Male & $323(59.3)$ & $465(50.9)$ & \multirow{2}{*}{$\begin{array}{c}\chi^{2}{ }_{1}=7.88 \\
p=0.013\end{array}$} \\
\hline Female & $159(40.7)$ & $449(49.1)$ & \\
\hline \multicolumn{4}{|c|}{ Age group } \\
\hline$<14$ & $158(40.4)$ & $425(46.5)$ & \multirow{2}{*}{$\begin{array}{l}\chi^{2}{ }_{1}=4.11 \\
p=0.089\end{array}$} \\
\hline$>14$ & $233(59.6)$ & $489(53.5)$ & \\
\hline \multicolumn{4}{|c|}{ Single Child } \\
\hline Yes & $174(44.5)$ & $421(46.1)$ & \multirow{2}{*}{$\begin{array}{c}x^{2}{ }_{1}=0.27 \\
p=0.636\end{array}$} \\
\hline No & $217(55.5)$ & $493(53.9)$ & \\
\hline
\end{tabular}




\section{Familial variables}

Family structure

Living with both biological parents

$334(85.4)$

$57(14.6)$

$808(88.4)$

$x^{2}{ }_{1}=2.26$

Others

$106(11.6)$

$\mathrm{p}=0.196$

Father's education level

University or above

$47(12.0)$

$91(10.0)$

High school/technical college

$102(26.1)$

207 (22.7)

$164(41.9)$

$436(47.7)$

$78(20.0)$

$180(19.7)$

Primary school or below

Mother's education level

University or above

High school/technical college

Middle school

$42(10.7)$

$78(20.0)$

$182(46.6)$

$89(22.8)$

Primary school or below

\section{Health Behaviours}

Smoking status

Current/Ex-smoker

Never

$372(95.1)$

Drank alcohol

Yes

No

$178(45.5)$

$213(54.5)$

$275(70.3)$

$116(29.7)$

No

Physically inactive

Yes

Parental health problems

Parental drinking problem

Yes

No

$97(24.8)$

$294(75.2)$

Parental gambling problem

Yes

No

$46(11.8)$

$345(88.2)$

$252(64.5)$

$98(25.1)$

Either one was ill

\section{Health literacy level}

High

\section{Competency of health information seeking}

Moderate/low

Online

$12.6(0.13)$

$11.2(0.16)$

$208(53.2)$

$183(46.8)$

Offline
65 (7.1)

168 (18.4)

459 (50.2)

222 (24.3) $x_{3}^{2}=4.46$

$\mathrm{p}=0.362$

$x_{3}^{2}=5.78$

$\mathrm{p}=0.231$

\begin{tabular}{c|c}
$80(8.8)$ & $\chi^{2}{ }_{1}=5.92$ \\
$834(91.3)$ & $p=0.036$
\end{tabular}

\begin{tabular}{c|c}
$373(40.8)$ & $\chi^{2}=2.50$ \\
$541(59.2)$ & $p=0.162$
\end{tabular}

541 (59.2)

$\mathrm{p}=0.162$

$719(78.7)$

$\chi^{2}{ }_{1}=10.48$

195 (21.3)

$\mathrm{p}=0.008$

$\chi^{2}{ }_{1}=5.73$

$\mathrm{p}=0.031$

627 (68.6)

140 (15.3)

$x^{2}{ }_{1}=2.83$

774 (84.7)

$\mathrm{p}=0.166$

$574(62.8)$

$\chi^{2}{ }_{2}=0.32$

240 (26.3)

$\mathrm{p}=0.828$

\begin{tabular}{l|l}
$420(46.0)$ & $x^{2}{ }_{1}=5.76$ \\
$494(54.0)$ & $p=0.069$
\end{tabular}

$x_{1}^{2}=5.76$
$p=0.069$

$12.0(0.15)$

$F_{(1,32)}=10.64$

$p=0.003$

$F_{(1,32)}=8.33$

$p=0.007$

*adjusted for cluster sampling effect 
competency of health information acquisition was significantly associated with intention for active health behaviour. There was a significant difference in the mean online CHIAS scores between those who had intended active health behaviour (mean $=12.6$, s.e. $=0.13$ ) and those who opted for other non-active behaviour (mean $=12.0$, s.e. $=0.15$ ) $(F(1,32)=10.64, p=0.003)$. Similar differences were also observed for the offline CHIAS scores with respondents who had intended active health behaviour scoring significantly higher than the other group with means of $11.2($ s.e. $=0.16)$ and 10.7 (s.e. $=0.12)$ respectively $(F(1,32)=8.33, p=0.007)$. Of the variables of interest, there were unadjusted associations between sex, smoking behaviour, physical inactivity, parental drinking and intention for active health behaviour $(p<0.050)$. These variables were included in further analyses.

Separate logistic regression analyses were conducted for the relationships between online, as well as offline competency of health information acquisition, and behavioural intent. The results obtained were presented in Tables 2 and 3. The results indicated that competency of health infor-

Table 2. Results obtained on the final model of the logistic regression analysis on the relationship between of online competency of health information acquisition and intention for active health behaviour.

\begin{tabular}{|l|c|c|}
\hline Variables & OR $(\mathbf{9 5 \%} \mathbf{C . I}) *$ & Significance \\
\hline Online competency & $1.06(1.01-1.12)$ & $\begin{array}{c}\mathrm{t}_{23}=2.40 \\
\mathrm{p}=0.022\end{array}$ \\
\hline Being a male & $1.50(1.15-1.95)$ & $\begin{array}{c}\mathrm{t}_{23}=3.09 \\
\mathrm{p}=0.004\end{array}$ \\
\hline $\begin{array}{l}\text { Current smoker } \\
\text { and ex-smoker }\end{array}$ & $0.49(0.26-0.92)$ & $\begin{array}{c}\mathrm{t}_{23}=-2.31 \\
\mathrm{p}=0.028\end{array}$ \\
\hline $\begin{array}{l}\mathrm{t}_{23}=-2.25 \\
\mathrm{p}=0.032\end{array}$
\end{tabular}

Table 3. Results obtained on the final model of the logistic regression analysis on the relationship between of offline competency of health information acquisition and intention for active health behaviour.

\begin{tabular}{|c|c|c|}
\hline & OR $(95 \%$ C.I)* & Significance \\
\hline \multicolumn{3}{|l|}{ Variables } \\
\hline Offline competency & $1.08(1.02-1.18)$ & $\begin{array}{l}t_{23}=2.94 \\
p=0.006\end{array}$ \\
\hline Being a male & $1.57(1.21-2.05)$ & $\begin{array}{l}t_{23}=3.51 \\
p=0.001\end{array}$ \\
\hline $\begin{array}{l}\text { Current smoker } \\
\text { and ex-smoker }\end{array}$ & $0.46(0.25-0.86)$ & $\begin{array}{c}t_{23}=-2.54 \\
p=0.016\end{array}$ \\
\hline Physically inactive & $0.68(0.49-0.95)$ & $\begin{array}{c}t_{23}=-2.36 \\
p=0.025\end{array}$ \\
\hline Parental drinking & $0.74(0.54-1.02)$ & $\begin{array}{l}t_{23}=-1.92 \\
p=0.064\end{array}$ \\
\hline
\end{tabular}

mation acquisition, both online and offline, was significantly associated with intention for active health behaviour among children after adjusting for potential confounders and the sample cluster effect. The odds of intention for active health behaviour increased by $6 \%$ for an increase in each unit of the online CHIAS score $(O R=1.06$, $95 \%$ C.I. $=1.01-1.12$ ). There was also a significant increase in the odds of the intention for active health behaviour by $8 \%$ for an increase in each unit of the offline CHIAS score (OR=1.08, 95\% C.I.=1.02-1.18). No significant interaction effects between any potential confounding factors were observed in the analyses.

\section{Discussion}

This study aims to examine the relationship between competency in health information acquisition, both online and offline, and the intention of active health behaviour among children in a large city. The results suggest that children's competency in health information acquisition, both online and offline, are significantly associated with their 
intention for active health behaviour. These are positive relationships with an increased competency in health information acquisition resulting in a corresponding increase in the likelihood of active health behavioural intent. An extensive search of the English language literature did not yielded a single report in a similar area of study. Hence, these results could be considered as a unique finding in the area of child health behaviour studies. Owing to the lack of other studies in this area, comparison of results is difficult.

The results of this study could potentially provide some interesting insight to the theorisation of the relationship between knowledge and skills of health behaviour and the intention for the health behaviour, particularly in children. While acknowledging that knowledge and skills are important determinants of actual health behaviour via their effect on the behavioural intent, as suggested in the Integrated Behavioural Model, the conceptualisation of the capacity to perform the behaviour requires an extension to include competency in information acquisition. [1] The results of the study indicate that competency may also play a role in the formation of the behavioural intent. As shown, a higher level of competency in health information acquisition, both online and offline, could result in a higher likelihood of behavioural intent. Should this be the case, the next question would be the underlying mechanisms between competency in heath information acquisition and the formation of behavioural intent. Would the relationship between behavioural intent and competency be due to an increased opportunities for gaining knowledge and skills or via some other mechanism? On the other hand the relationship between competency in health information acquisition and the intention for active health behaviour may not be a unidirectional one. It could be possible that, for certain personal reasons, children with a higher level of behavioural intent could build up their competency in health information acquisition via a greater involvement in active information seeking behaviour. These would be worthy directions for future research.

The results obtained from this study have a direct implication on child health promotion and education in many areas such as risky health behaviours, obesity, diabetes, and mental health. Competency is a malleable attribute that can be enhanced with appropriate measures such as training. [13] The effectiveness of the competency-based training model on knowledge, skills, and attitude has also been demonstrated in many different disciplines. $[20,21]$ Competency in health information acquisition among children and young people can also be enhanced by applying the training model to improve their attitudes, information and acquisition seeking skills. Moreover, skills for information acquisition could be transferred across different disciplines and health problems. Hence the competency of health information acquisition could be built up across many different health issues in a relatively effortless manner. This training could also be incorporated as part of existing school health programs, such as the Healthy School Program which was designed in the US and implemented in the primary and secondary schools of many countries. [22]

There are strengths and weaknesses in this study. This is a population-based study that includes a random sample of students from two large cities utilising a two-stage cluster random sampling technique. An appropriate statistical analytical approach has been used to adjust for the effect of cluster sampling. The use of a standardised and validated assessment instrument for competency in health information acquisition minimised some measurement biases. Some potential limitations have also been identified in this study. For example, a cross-sectional study could be considered as an appropriate design for exploring the potential relationship between the exposure or study factor and the outcome. However, the 
evidence provided from such a study can only be considered as associative and is insufficient to draw any causal inference. [23] This study can be considered as an exploratory study to identify the potential association between competency in health information acquisition and the intention for active health behaviour in children. Some potential confounding factors of behavioural intent suggested by the Integrated Behavioural Model, such as attitude towards active health behaviour, personal agency, and particularly perceived norm, were not included in this study. Future studies could be conducted with the inclusion of these variables. Additionally a different study design, design such as a longitudinal cohort study, could elucidate whether the association is of a causal nature and should be implemented.

\section{References}

1. Montano DE, Kazprzyk D. Theory of Reasoned Action, Theory of Planned Behaviour, and the Integrated Behavioural Model. In: Glanz K, Rimer BK, Viswanath K (eds) Health Behaviour and Health Education. Theory, Research, and Practice, $4^{\text {th }}$ ed. Jossey-Bass, San Francisco, 2008, pp 67-92.

2. Jaccard J, Dodge T, Dittus P.Parent-adolescent communication about sex and birth control: A Conceptual frame work. In: Feldman S, Rosenthal DA (eds) Talking Sexuality: ParentAdolescents Communication. New Directions in Child and Adolescent Development. No 97, Jossey-Bass, San Francisco, 2002, pp 9-41.

3. Godin G, Germain M. Predicting first lifetime plasma donation among whole blood donors. Transfusion. 2013; 53 Suppl 5: 157S-61S.

4. Hall ME, Bergman RJ, Nivens S. Worksite health promotion program participation: a study to examine the determinants of participation. Health Promot Pract. 2013; Epub ahead of print.

5. Hamade H, Chaaya M, Saliba M, Chaaban R, Osman H. Determinants of exclusive breastfeeding in an urban population of primiparas in Lebanon: a cross-sectional study. BMC Public Health. 2013; 13:702.

6. Iqbal U, Ho CH, Li YC, Nguyen PA, Jian WS, Wen HC. The relationship between usage intention and adoption of electronic health records at primary care clinics. Comput Methods Programs Biomed. 2013; 112:731-7.

7. Tzilos GK, Strong DR, Abrantes AM, Ramsey SE, Brown RA. Quit intention as a predictor of quit attempts over time in adolescents with psychiatric disorders. Am J Addict. 2013; Epub ahead of print.

8. Wood KV, Ogden J. Predictors of decreased binge eating following laparoscopic adjustable gastric banding using the Health Action Process Approach model. Psychol Health Med. 2013; Epub ahead of print.

9. Wu DF, Hu J, Zhang ZY, Wang L. Evaluation of an HIV-related behavior change project for female sex workers in Central China. Chin Med J (Engl). 2013; 126: 2451-8.

10. Armitage CJ, Rowe R, Arden MA, Harris PR. A Brief Psychological Intervention that reduces adolescent alcohol consumption. J Consult Clin Psychol. 2014; 82: 546-50.

11. Park SE, Lee K, Yun SN, Cui W. Structural model of factors influencing smoking behavior among Korean-Chinese adolescent boys. App/ Nurs Res. 2014; S0897-1897: 00030-5.

12. Tapscott D. Educating the Net Generation. Educ Leadersh, 1999; 56: 7-11.

13. Dunois D. The competency casebook. International Society for Performance Improvement, Amherst, MA: HRD, \& Silver Spring MD. 1998.

14. Teodorescu T. Competence versus competency: what is the difference? Performance Improvement. 2006; 10. 
15. Norman C, Skinner HA. eHEALS: The eHealth Literacy Scale. J Med Internet Res. 2006; 8: e27.

16. World Health Organization. Global recommendations on physical activity for health. World Health Organization, Geneva, 2010.

17. Chisolm DJ, Buchanan L. Measuring adolescent Functional Health Literacy: a pilot validation of the test of Functional Health Literacy in adults. J Adol Health. 2007; 41: 312-4.

18. Chang L, Hsieh P, Liu C. Psychometric evaluation of the Chinese version of the short- form Test of Functional Health Literacy in adolescents. J Clinic Nursing. 2012; 21: 2429-37.

19. Chang L. Health literacy, self-reported status and health promoting behaviours for adolescents in Taiwan. I Clinic Nursing. 2010; 20: 190-6.

20. Donovan RA, Ponce AN (2009) Identification and measurement of core competencies in professional psychology: Areas for consideration. Training and Education in Professional Psychology. 2009; 3 Suppl: S46-S49.

21. Hoge MA, Paris $M$, Adger $H$, Collins FL, Finn CV, Fricks LG, et al. Workforce competencies in Behavioral Health: An Overview. Adm Policy Ment Health. 2005; 32:593-631.

22. Alliance for a Healthier Generation. http://www. healthiergeneration.org/. Accessed on 19th March 2014.

23. Rothman KJ, Greenland S. Morden Epidemiology 2nd Ed. Lippincott Williams \& Wilkins, Philadelphia. 1998.

\section{Comment on this article:}

AB in $8+\mathbf{S} P$ T1

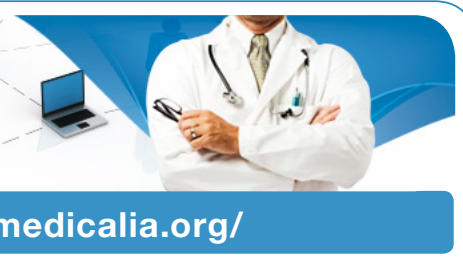

http://medicalia.org/

Where Doctors exchange clinical experiences, review their cases and share clinical knowledge. You can also access lots of medical publications for free. Join Now!

\section{Publish with iMedPub}

http://www.imed.pub

International Archives of Medicine is an open access journal publishing articles encompassing all aspects of medical science and clinical practice. IAM is considered a megajournal with independent sections on all areas of medicine. IAM is a really international journal with authors and board members from all around the world. The journal is widely indexed and classified Q1 in category Medicine. 